\section{Promouvoir la FMH?}

Dr Claude Aubert, Membre du CC

La promotion de la FMH se heurte d'emblée à la question de la cible. S'il devait y avoir une action, une publication, une exhortation, quel serait le public auquel nous nous adresserions?

Vu de l'extérieur, la FMH est un lobby puissant, mais ses membres (du moins la base) n'en sont pas forcément conscients, puisqu'ils critiquent fréquemment le silence et l'inertie de leur Fédération. Travail dans l'ombre ou sieste à l'ombre?

Il est bien différent de faire la promotion d'un Tigre de papier ou d'une forteresse à la Vauban.

L'écrasante majorité des médecins font partie de la FMH, "automatiquement», mais cette participation n'est pas synonyme d'engagement. Il est difficile de savoir si l'on doit se baser sur ce qui est proclamé ("Ils ne font rien!») ou sur ce qui est démontré, le paiement des cotisations étant, à cet égard, un geste significatif, en l'occurrence très réconfortant.

Promouvoir un militantisme en brusquant un engagement personnel? Se tromper quant à l'état d'esprit des troupes en confondant ce qui est dit et ce qui est montré?

La FMH repose statutairement sur des organisations de base, qui ont leur propre politique, leurs propres intérêts. La FMH est-elle plus que la somme de ses composants? Ou constitue-t-elle une "menace» que chacun brandit pour s'appuyer sur une puissance virtuelle ("La FMH a dit que ...»), quitte à s'en désolidariser le moment venu?
Promouvoir la FMH, est-ce d'abord intervenir auprès des Sociétés cantonales de médecine (SCM), des Sociétés de discipline médicale (SDM), de l'Association des médecins principaux des hôpitaux de Suisse (AMPHS) et de l'Association des médecins assistants et chefs de clinique (ASMAC), avant même d'atteindre à leur cabinet de consultation le Docteur ou la Doctoresse Trucmuche?

La population, depuis des siècles, a la même vision dichotomique de la profession. Grosso modo, «notre propre médecin est le meilleur, mais les autres, alors ..."

Promouvoir la FMH, est-ce discourir de la médecine, plus exactement la médecine en tant que science ou faire l'éloge des médecins, plus exactement de ces hommes et de ces femmes qui exercent cette profession? Est-ce promouvoir la profession au sein des autres professions "parentes ou alliées» (comme cela se dit dans les avis de décès)? Ou encore, est-ce promouvoir une philosophie de la vie synthétisée par Hippocrate?

N'oublions pas les puissants, ceux qui légifèrent et qui gouvernent. La lutte contre la maladie n'est pas forcément une préoccupation désintéressée. Au gré du politiquement correct, la médecine et la santé se posent successivement comme objectifs prioritaires. A moins que l'état des finances publiques ne contraigne à économiser dans ce secteur, pour regarnir l'escarcelle de plus chanceux quémandeurs.

Promouvoir la FMH, est-ce évoluer dans la cour des grands pour attirer leur bienveillante attention, ou militer dans l'arrière-cour des menus, pour soutenir leur dignité?

Questions, questions. On peut toujours poser des questions.

Lecteur, lectrices, qu'en pensez-vous? 